\title{
Minimização da Intervenção Humana para Detectar e Solucionar Anomalias Rede de Computadores
}

\author{
Alexandre Amaral ${ }^{1}$, Ana Paula Malheiro ${ }^{2}$ \\ ${ }^{1}$ Instituto Federal de Santa Catarina (IFSC) \\ CEP 89254-430 - Jaraguá do Sul - SC - Brasil \\ ${ }^{2}$ Instituto Federal Catarinense (IFC) \\ Caixa Postal 2016 - Camboriú - SC - Brasil \\ alexandre.amaral@ifsc.edu.br, anapaula@ifc-camboriu.edu.br
}

\begin{abstract}
This paper proposes a system to detect and apply corrective actions when anomalous events occur in the network. Management goals are defined through the metrics and the self-healing property of the autonomic computing is used, empowering the system to perform corrective actions without human intervention. NEMES (Network Metric Specification) a domain-specific language was developed to build the metrics. The system uses IP flows to reduce the volume of data to be processed, allowing its use in large-scale networks. Tests performed in a real environment have shown the effectiveness and potential of the proposed system to assist in the network management.
\end{abstract}

Resumo. Neste trabalho é proposto um sistema para detectar e aplicar ações corretivas na ocorrência de eventos anômalos na rede. Os objetivos da gerência são definidos através de métricas e a propriedade de autorreparo da computação autonômica é utilizada para que o sistema execute ações corretivas sem a intervenção humana. NEMES (Network Metric Specification), uma linguagem de domínio específico foi desenvolvida para a escrita das métricas. O sistema utiliza fluxos IP que reduz o volume de dados a serem processados, permitindo sua utilização em redes de grande escala. Testes realizados em um ambiente real demonstraram a eficácia e o potencial do sistema proposto para auxiliar no gerenciamento de rede.

\section{Introdução}

Esforços têm sido realizados pela comunidade cientifica a fim de desenvolver mecanismos que auxiliem no monitoramento e na segurança da rede. Algumas soluções do tipo NIDS (Network-based Intrusion Detection System) têm sido propostas. Porém, [Bhuyan et al. 2014] apresentaram recentemente diversos fatores que limitam a aplicação desses mecanismos para funcionamento em tempo real em redes de grande escala. A inspeção de todos os pacotes transmitidos pela rede requer um alto processamento e espaço de armazenamento. Os alarmes emitidos na ocorrência de um evento anômalo requer uma análise manual do administrador de rede para que o problema seja reparado. A aplicação dos NIDS em um escopo mais amplo de gerenciamento não tem sido endereçada. Isto inclui o monitoramento de serviços e usuários, identificação de pontos de gargalo na rede, além de não fornecerem um meio para definição e monitoramento de KPIs (Key Performance Indicator) específicos, como uptime/downtime de um recurso de rede. 
Nesse trabalho é apresentado um sistema para detectar eventos anômalos na rede e aplicar ações corretivas, com funcionamento em tempo real. Os objetivos da gerência são definidos através de métricas, e o sistema aplica a propriedade de autorreparo da computação autonômica [Magalhães e Silva 2013], a fim de que as ações corretivas e/ou preventivas sejam executadas sem a intervenção humana. NEMES (Network Metric Specification), uma linguagem de domínio específico foi desenvolvida para a escrita das métricas. Diferentemente das propostas que utilizam como fonte de dados os pacotes IP, o sistema proposto utiliza os fluxos IP. Esta escolha visa reduzir o volume de dados a serem processados e a aplicação do sistema em redes de grande escala. Testes realizados em um ambiente real demonstraram a eficácia e o potencial desse sistema para auxiliar no gerenciamento de rede.

\section{Sistema Proposto}

A Figura 1 apresenta os principais componentes do sistema. Os fluxos IP coletados dos dispositivos ativos na rede são processados pelo $F E$ (Feature extractor). O FE obtêm as propriedades dos fluxos (e.g., endereço IP de origem, número de pacotes) enviando os para o componente $M E$ (Metric processor). Utilizando as métricas lidas da $K B$ (Knowledge base), as propriedades dos fluxos são analisadas pelo $M E$, com o objetivo de identificar os fluxos anômalos ou indesejáveis. As métricas contêm as condições das propriedades dos fluxos considerados anômalos e as ações que devem ser executadas caso eles ocorram na rede. Quando um fluxo é considerado anômalo a ação especificada na métrica é executada pelo componente AP (Action processor), e o resultado de sua execução é armazenado na $K B$.

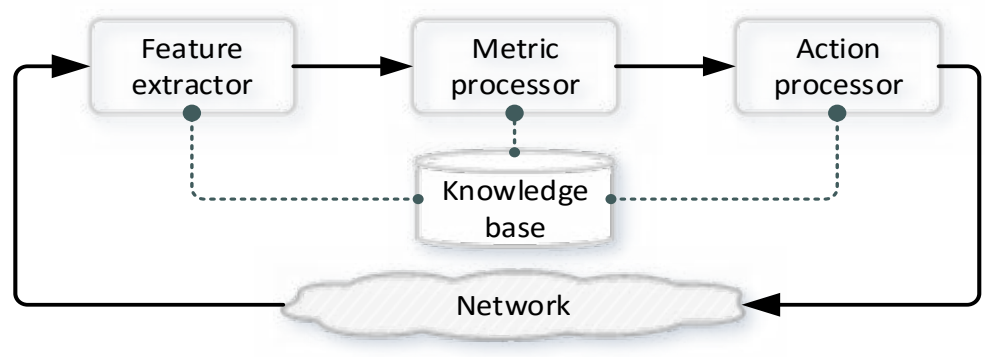

Figura 1. Principais componentes do sistema.

Neste trabalho, uma métrica é uma condição definida pelo administrador que caso ela ocorra na rede uma ação é disparada. A Figura 2 apresenta uma métrica para detecção de um ataque Ping of Death. Este ataque consiste no envio de fluxos ICMP com pacotes maiores do que 65535 bytes. Tais características são definidas na condition da métrica. $\mathrm{Na}$ ocorrência desse ataque, a métrica especifica duas ações a serem realizadas: execute e notify. O método execute aplica uma ação corretiva e/ou preventiva. No exemplo, um script será executado com o propósito de bloquear o ataque. Simultaneamente, uma notificação será enviada para o administrador de rede através do método notify. O meio de comunicação utilizado no exemplo é o e-mail. Entretanto, o sistema está sendo desenvolvido como uma interface que permita a utilização de outros meios, tais como SMS (Short Message Service) e log. 


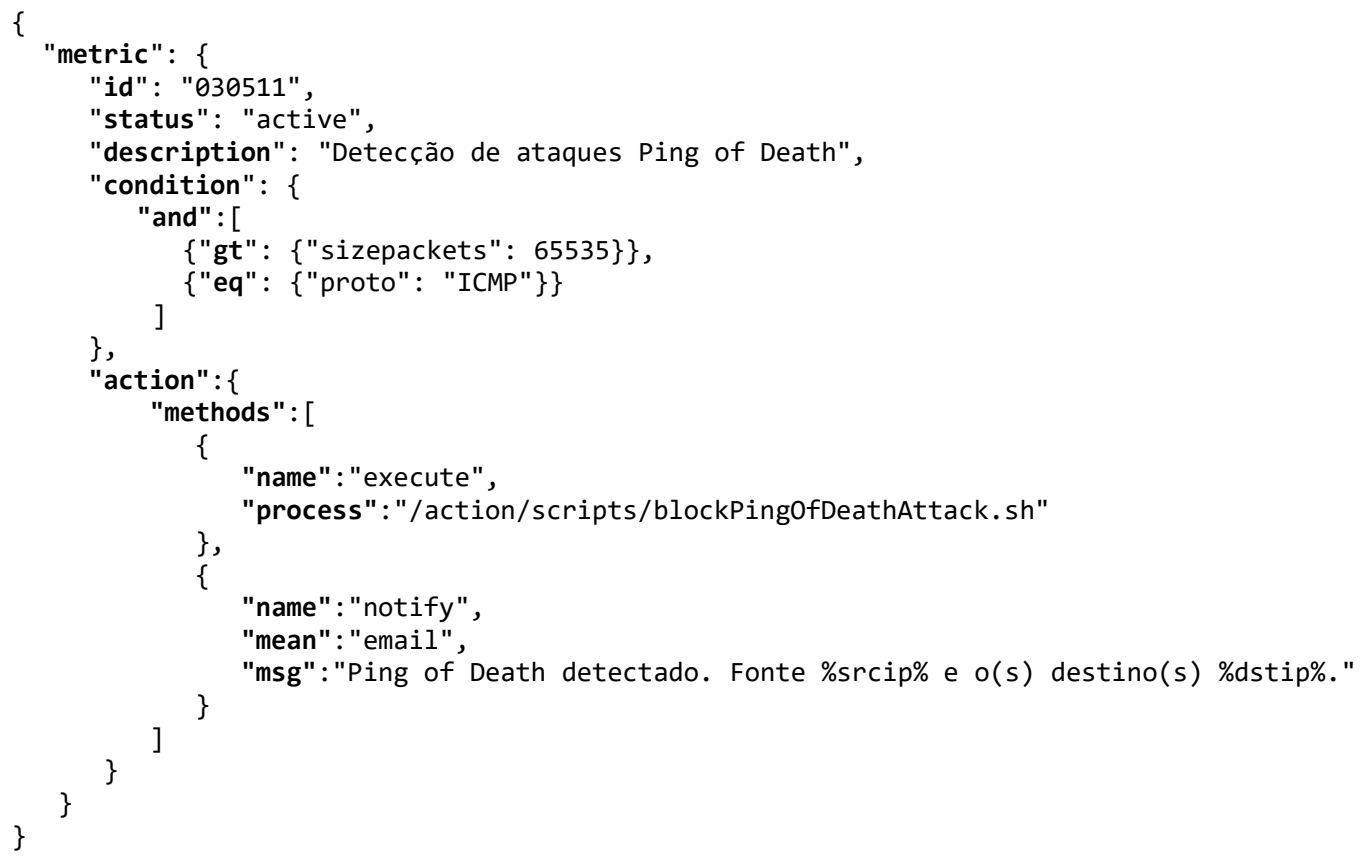

Figura 2. Exemplo de uma métrica para detectar um ataque Ping of Death.

Uma linguagem de domínio específico, denominada NEMES (Network Metric Specification) foi desenvolvida para a criação das métricas. NEMES utiliza a notação JSON como mostra a Figura 2. A escolha do JSON dentre outras linguagens (e.g., $\mathrm{XML}$ ), se por algumas razões. O JSON é mais leve, mais fácil para leitura e escrita por humanos e por estar sendo amplamente utilizado nos últimos anos [Yang 2012]. Antes de serem disponibilizadas na base de conhecimento, as métricas escritas pelo administrador de rede são processadas pelo compilador NEMES, desenvolvido com o propósito de realizar a análise léxica, análise sintática e análise semântica.

\subsection{Aplicação do autorreparo}

Quando a condição de uma métrica é atendida o componente AP (Action Processor) é acionado. Através deste componente, o sistema proposto implementa o método execute com o objetivo de aplicar a propriedade de autorreparo da computação autonômica. No contexto do gerenciamento de redes, uma gama de ações pode ser definida pelo administrador para fins de autorreparo. O bloqueio de acesso e a finalização de uma sessão de usuário pelo abuso dos recursos de um servidor são procedimentos que podem ser especificados, a fim de que os serviços prestados pela rede continuem operando sem danos.

\section{Estudo de Caso}

Testes em um ambiente real de rede foram realizados com o propósito de validar o sistema proposto. Para este fim, um testbed foi montado, como ilustra a Figura 3. Fluxos IP são coletados do gateway/firewall através do protocolo NetFlow v9 a cada cinco minutos. O sistema foi desenvolvido com a linguagem Java. Para a base de conhecimento foi utilizada o banco de dados Neo4J [Neo4J 2016]. 


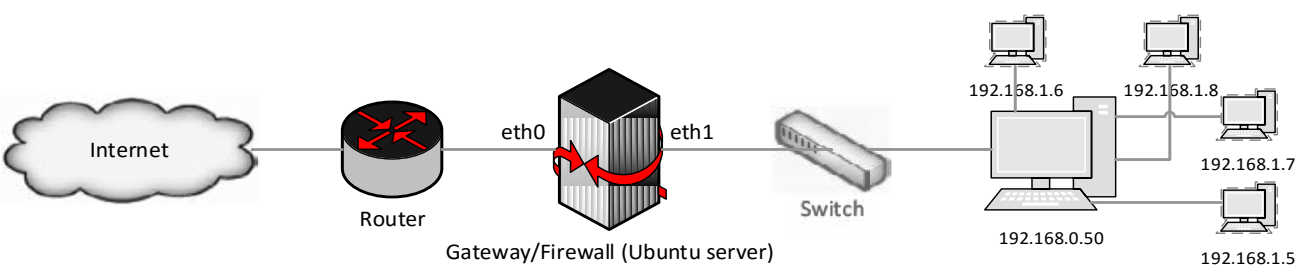

Figura 3. Testbed para realização dos testes.

Nos experimentos, as métricas foram criadas para a detecção de Land attack, Smurf attack, detecção de acesso não permitido a diversos sites proibidos pela instituição, incluindo redes sociais e de conteúdo adulto. Para geração dos ataques Land attack e Smurf attack foi utilizada a ferramenta Hayane [Hyenae 2016] lançados a partir das máquinas virtuais hospedadas na máquina 192.168.0.50. Para aplicação das ações de autorreparo foi utilizado o firewall Iptables instalado na máquina Gateway/Firewall.

Os testes realizados demonstraram a eficácia do sistema para detectar e aplicar as correções especificadas nas métricas. As ações testadas correspondem ao método execute e notify. Em todos os testes foram utilizados scripts para bloquear os fluxos indesejados via firewall. O método notify foi testado utilizando o e-mail e o armazenamento textual do resultado da aplicação da ação em arquivos de $\log$.

\section{Conclusão}

Nesse trabalho foi apresentado um sistema para detecção de atividades anômalas na rede. Através de uma linguagem de domínio específica denominada NEMES, métricas podem ser escritas para que o sistema identifique atividades que vão além dos ataques e ações maliciosas endereçadas pelas soluções atuais. A utilização da propriedade de autorreparo da computação autonômica permite que o sistema monitore, detecte as ações especificadas pelas métricas e execute ações para solucioná-las. Os testes demonstraram a capacidade do sistema de tomar ações sem a intervenção humana, auxiliando o administrador na árdua tarefa do gerenciamento de rede. Como trabalhos futuros, desejamos explorar outras propriedades da computação autonômica, realizar mais testes e utilizar o sistema de forma distribuída.

\section{Referências}

Bhuyan, M.H. Bhattacharyya, D.K. e Kalita, J.K. (2014) "Network Anomaly Detection: Methods, Systems and Tools". Communications Surveys \& Tutorials, IEEE, vol.16, no.1, p. 303-336, 2014.

Hyenae. Disponível em: http://sourceforge.net/projects/hyenae/

Magalhães J. P. e Silva L. M., "Self-healing Performance Anomalies in Web-based Applications," Network Computing and Applications (NCA), 2013 12th IEEE International Symposium on, Cambridge, MA, pp. 81-88, 2013.

Neo4j. "The World's Leading Graph Database”. Disponível em: http://www.neo4j.org/

Yang Y. "Impact data-exchange based on XML", Computer Science \& Education (ICCSE), 7th International Conference. p.1147-1149, 2012. 